\title{
Influence of anterior chamber depth on postoperative refractive outcome in Chinese eyes
}

\author{
Rachel SH Wong' ${ }^{1}$, Keith Ong ${ }^{2,3}$ \\ 'Discipline of Clinical Ophthalmology \& Eye Health, University of Sydney, Sydney, \\ Australia; ${ }^{2}$ Royal North Shore Hospital Department of Ophthalmology, University \\ of Sydney, Sydney, Australia; ${ }^{3}$ Dalcross Adventist Hospital, Sydney, Australia
}

\begin{abstract}
Aim or Purpose: This study aims to evaluate the refractive surprise (RS) after cataract surgery with various intraocular lens (IOL) formulas in eyes with very shallow or deep anterior chamber depth (ACD).

Design: This is a prospective cohort study of patients from a private ophthalmology practice in Sydney.

Methods: Thirty-one patients who had their cataract surgery in 2014 were included. The cohort consists of 20 eyes with ACD $<2.8 \mathrm{~mm}$ and 25 eyes with ACD $>3.2 \mathrm{~mm}$. Patients' demographic variables and their predicted refractive outcomes using the SRK-T, Haigis, Holladay 1, and Holladay 2 IOL formulas were collected. Actual refractive outcomes were obtained from consultations at least one-month postoperatively. RS was calculated from the difference between predicted refraction outcome of IOL formulas and the actual postoperative refraction achieved.

Results: The linear correlations between ACD and RS were not significant ( $p>0.05)$. In the group with $A C D<2.8 \mathrm{~mm}$, the mean refractive surprise using SRK-T, Haigis, Holladay 1 , and Holladay 2 formulas were $-0.191 \pm 0.541,-0.189 \pm 0.444,-0.201 \pm 0.449$, and -0.154 $\pm 0.489 \mathrm{D}$, respectively. In the group with $A C D>3.2 \mathrm{~mm}$, the mean refractive surprise using the IOL formulas were $-1.364 \pm 0.541,-1.420 \pm 0.541,0.027 \pm 0.394$, and $-0.045 \pm$ $0.343 D$, respectively.
\end{abstract}

Conclusion: The positive linear correlation between ACD and RS was weak. In eyes with $A C D<2.8 \mathrm{~mm}$, the least RS was found with the Holladay 2 formula, while in eyes with $A C D>3.2 \mathrm{~mm}$, this was found with Holladay 1.

Keywords: anterior chamber depth, cataract surgery, intraocular lens formula, refractive outcome, refractive surprise

\section{Introduction}

Refractive outcome is a major factor in cataract surgery. In our modern era, a critical advancement is in the ability to estimate the power of the intraocular lens (IOL) that is to be implanted. ${ }^{1}$ The power of IOL needed to produce the desired postoperative refraction outcome is determined by theoretical formulas such

Correspondence: Dr. Keith Ong, 2 Railway Ave, Eastwood NSW 2122, Australia.

E-mail: keithong@optusnet.com.au 
as SRK/T (Sanders-Retzlaff-Kraff)/Theoretical), Haigis, and Holladay, prior to the cataract extraction. ${ }^{2}$ Accurate biometric measurements of the eye and use of appropriate calculations have allowed surgeons to improve the predictability of refractive outcomes.

The parameter for diagnosing a shallow or deep eye is based on anterior chamber depth (ACD), ${ }^{3}$ which represents the anatomical distance between the corneal endothelium and the anterior capsule of the crystalline lens. ${ }^{4}$ In optical biometry, ACD is calculated from the corneal epithelium to the lens apex. Older mathematical formulas like SRK/T are determined by measurements of axial length $(A L)$ and corneal curvature $(K)$, with $A C D$ as a surrogate that is estimated from keratometry; newer IOL implant power formulas like Haigis often involve measuring preoperative ACD. ${ }^{1}$ The newer formulas theoretically yield lower prediction errors. This is because ACD is used to predict the postoperative effective lens position (ELP), which influences the postoperative refraction. However, refractive surprise (RS) can occur after surgery even if the modern formulae are used, especially for those with extreme myopia and extreme hypermetropia (i.e., very large or very small eyes). It is known that IOL power calculations tend to yield hypermetropic refractive prediction errors in long eyes (large AL). ${ }^{5}$ Therefore, RS are often anticipated for such extreme cases and targeting a bit of residual myopia ${ }^{6}$ would help prevent hypermetropia post-cataract surgery.

The purpose of our study is to evaluate the influence of ACD on postoperative refractive outcomes by evaluating the RS of various IOL formulas after cataract surgery, comparing eyes with very shallow and very deep ACD.

\section{Methods}

In this prospective cohort study, 31 patients who had their cataract surgery with IOL implantation from January to December 2014 were included. Patients included are of Asian ethnicity and had attended a private ophthalmology clinical practice in Sydney, NSW, Australia. The cohort consists of 20 eyes with ACD less than $2.8 \mathrm{~mm}$ and 25 eyes with ACD greater than $3.2 \mathrm{~mm}$. Patients' demographic details and biometric measurements were collected using IOLMaster 500 (Carl Zeiss Meditec AG; Germany), through which preoperative keratometry, ACD, and IOL lens power calculations were obtained using Carl Zeiss IOLMaster Advanced Technology V.7.5 software. The refractive outcomes were obtained from postoperative consultations at least one-month after cataract surgery.

We then analysed the clinical variables with regard to refractive outcomes using the SRK-T, Haigis, Holladay 1, and Holladay 2 IOL formulas. RS was calculated as the difference between the predicted postoperative refractions of different IOL formulas and the actual postoperative refraction achieved. Analysis of RS was done using Excel software (Microsoft; United States). Linear regression analysis 
was performed using the method of least squares. A P value of less than 0.05 is considered significant; all tests were 2 -tailed. ${ }^{7}$

\section{Results}

In the patient group with 20 eyes with small ACD (ACD $<2.8 \mathrm{~mm})$, there were 13 patients with a mean age of $70 \pm 6.96$ years (55-86). Their average AL was $23.6 \pm$ $1.352 \mathrm{~mm}(21.58-27.54)$ and mean ACD of $2.69 \pm 0.117 \mathrm{~mm}$ (2.3-2.81). In the group with 25 eyes with large $A C D(A C D>3.2 \mathrm{~mm})$, there were 18 patients with a mean age of $68 \pm 5.91$ years (58-79). Their average AL was $25.8 \pm 1.28 \mathrm{~mm}$ (22.49-27.99) and mean ACD of $3.52 \pm 0.218 \mathrm{~mm}$ (3.21-4.07). Across 31 patients, ACD increased significantly with $A L$, with a linear correlation of $0.497(p<0.05)$ (Fig. 1). The

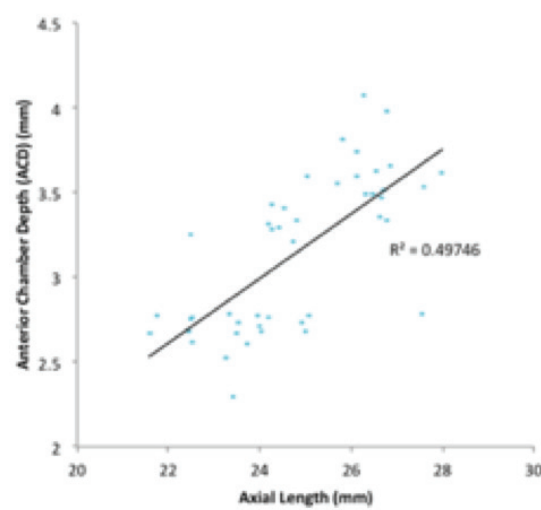

Fig 1. Relationship between AL and ACD in 45 Chinese eyes. Chinese eyes

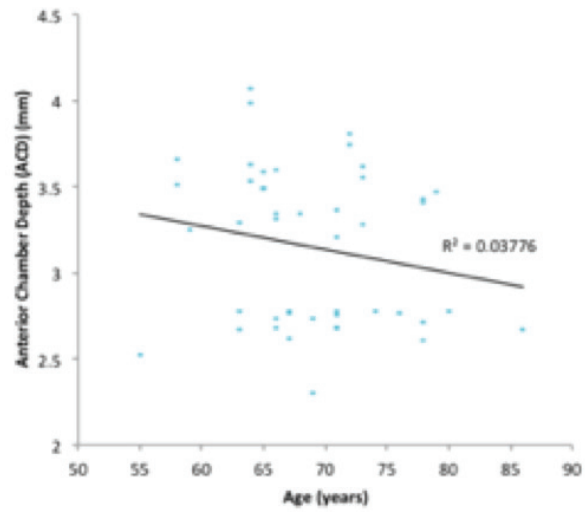

Fig 2. Relationship between age and ACD in 45 Chinese eyes.

\begin{tabular}{|c|c|c|c|c|c|c|c|c|}
\hline & Measure & $\begin{array}{l}\text { Age } \\
\text { (year) }\end{array}$ & $\begin{array}{l}\mathrm{IOL} \\
\text { used } \\
\text { (D) }\end{array}$ & $\begin{array}{l}\text { Preop } \\
\text { Refraction } \\
\text { (D) }\end{array}$ & $\begin{array}{l}\text { Postop } \\
\text { Refraction } \\
\text { (D) }\end{array}$ & $\begin{array}{l}\text { AL } \\
(\mathrm{mm})\end{array}$ & $K(D)$ & $\begin{array}{l}\text { ACD } \\
(\mathrm{mm})\end{array}$ \\
\hline \multirow{4}{*}{$\begin{array}{l}\text { Shallow } \\
\text { eyes } \\
(A C D< \\
2.8 \mathrm{~mm} \text { ) }\end{array}$} & Mean & 70 & 21.4 & -0.313 & -0.875 & 23.64 & 43.97 & 2.69 \\
\hline & SD & 6.96 & 4.07 & 2.73 & 0.524 & 1.352 & 1.648 & 0.117 \\
\hline & Min & 55 & 10 & -2.75 & -2.125 & 21.58 & 41.49 & 41.49 \\
\hline & Max & 80 & 28.5 & 2.75 & -0.25 & 27.54 & 46.59 & 46.59 \\
\hline \multirow{4}{*}{$\begin{array}{l}\text { Deep } \\
\text { eyes } \\
(\mathrm{ACD}< \\
3.2 \mathrm{~mm})\end{array}$} & Mean & 68 & 15.9 & -3.28 & -1.47 & 25.76 & 43.84 & 3.52 \\
\hline & SD & 5.91 & 3.85 & 3.97 & 0.519 & 1.282 & 1.204 & 0.218 \\
\hline & Min & 58 & 11 & -8.75 & -2.25 & 24.18 & 41.86 & 3.25 \\
\hline & Mean & 78 & 25.5 & 3.5 & -0.5 & 27.99 & 46.85 & 4.07 \\
\hline
\end{tabular}


Table 2. Mean postoperative RS using SRK-T, Haigis, Holladay 1, and Holladay 2 IOL formulas in 20 shallow (ACD $<2.8 \mathrm{~mm}$ ) and 25 deep (ACD $>3.2 \mathrm{~mm}$ ) Chinese eyes

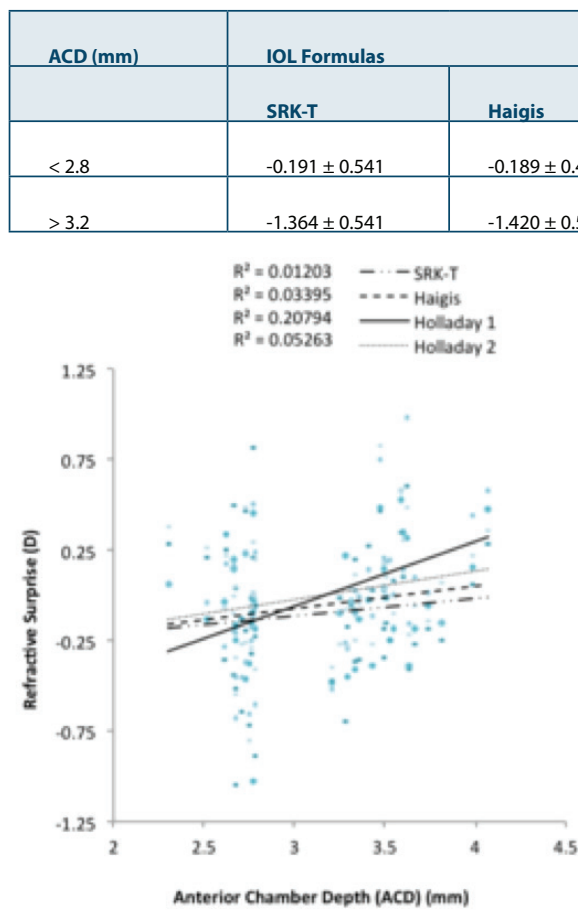

Fig 3. Relationship between postoperative RS and ACD in 45 Chinese eyes.

\begin{tabular}{|l|l|l|} 
& Holladay 1 & Holladay 2 \\
\hline 0.444 & $-0.201 \pm 0.449$ & $-0.154 \pm 0.489$ \\
\hline 0.541 & $0.027 \pm 0.394$ & $-0.045 \pm 0.343$ \\
\hline
\end{tabular}

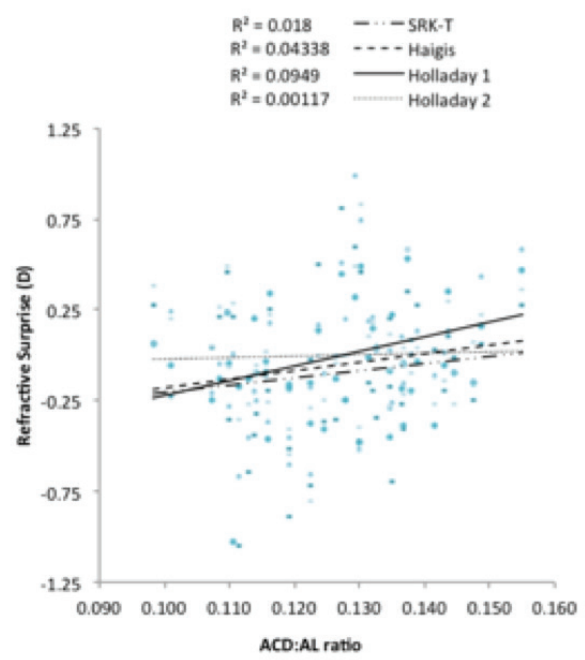

Fig 4. Relationship between $A C D: A L$ ratio and postoperative RS in 45 Chinese eyes

decrease in ACD with aging was not significant in our study population (Fig. 2).

In eyes with $A C D<2.8 \mathrm{~mm}$, the mean refraction was $-0.313 \pm 2.73 \mathrm{D}$ preoperatively and $-0.875 \pm 0.524 \mathrm{D}$ postoperatively ( $>1$-month) at follow-up (Table 1 ). The least RS was found with the Holladay 2 formula at $-0.154 \pm 0.489$. In eyes with ACD $>3.2 \mathrm{~mm}$, the mean refraction was $-3.28 \pm 3.97$ D preoperatively and $-1.47 \pm 0.519$ D postoperatively at follow-up (Table 2). The least RS was found with Holladay 1 at $0.027 \pm 0.394$ (Table 2). Overall, the increase in RS with increasing ACD was not significant. Linear regression analysis showed that the influence of ACD on RS was greatest using the Holladay 1 formula at R2 of 0.20794 ( $p>0.05$ ); the weakest correlation was attained using the SRK-T formula at R2 of $0.01203(p>0.05)$ (Fig. 3). Similarly, the correlations between the ACD:AL ratio and postoperative RS surprise using different IOL formulas were not significant, with $\mathrm{R} 2$ values of 0.018 , $0.04338,0.0949$, and 0.00117 with the SRK-T, Haigis, Holladay 1, and Holladay 2 formulas, respectively (Fig. 4). 


\section{Discussion}

The latest-generation IOL power prediction formulas have considerably improved refractive outcomes. The Holladay 1 and 2 and SRK/T formulas both use thin-lens theory, with different prediction algorithms for ELP and adjustment factors for short and long eyes; ${ }^{8}$ the Haigis formula is based on exact thick-lens theory and does not use any other adjustment factors. ${ }^{8}$ Nonetheless, there is still much controversy about which is significantly and consistently better. ${ }^{9}$ Our study has found that the least RS was found with the Holladay 2 formula in small eyes with $A C D<2.8 \mathrm{~mm}$ and Holladay 1 in large eyes with $A C D>3.2 \mathrm{~mm}$. This is consistent with other studies showing that the Holladay 2 formula should be used for patients with $\mathrm{AL}$ and corneal powers that are outside the normal ranges of 22-25 mm and $42-46 \mathrm{D} .^{2}$

The SRK-T formula can predict refraction in patients with normal AL and ACD $<3 \mathrm{~mm}$ with less error and is preferred over other formulas. ${ }^{10}$ However, the improved accuracy of prediction can also be a function of the surgeon's technical skills in implanting lenses consistently in a capsular bag. ${ }^{1}$ Furthermore, we also have to consider the range besides the mean of RS when comparing different formulas. It has been found that the use of preoperative ACD in combination with $A L$ for the precision of the pseudophakic $A C D$ can be expected to improve the accuracy of IOL power calculation. ${ }^{11}$ Our findings are consistent with their results, especially in Chinese eyes, which have more variable ACD.

Our results suggest that there is a tendency to have greater hypermetropic surprise in large ACD, as well as when ACD is disproportionately larger than AL. The Holladay 2 formula was found to have the least RS across all ACD:AL ratios, hence a practical suggestion would be to use the Holladay 2 formula for very shallow or deep eyes. Also, we know that $A C D$ varies with age ${ }^{12}$ as the lens thickens. However, ELP may not vary with age. ELP is known to influence the final refractive outcome and given there can be much variability in ELP among Chinese eyes, this can be a source of RS. Currently, ELP is estimated from AL and $\mathrm{K}$ readings. ${ }^{13}$ Perhaps, estimating the distance from the corneal epithelium to the middle of the cataract lens rather than to the anterior capsule provides a better ELP estimate. We note that although ACD is defined as the anatomical distance between the corneal endothelium and the anterior capsule of the crystalline lens, ${ }^{4}$ the IOLMaster 500 measures ACD as the distance from corneal apex to lens apex, hence including corneal thickness. ${ }^{14}$ Modifying this measurement by taking it from the corneal epithelium to the centre of the cataract lens may provide a more accurate estimate of ELP. This may be possible in newer optical biometry instruments with swept source optical coherence tomography technology, as corneal thickness and cataract lens thickness can be measured. Perhaps we could modify $\mathrm{IOL}$ formulas by replacing the "ACD" in the formulas to a modified parameter 
that can be called "estimated $E L P$ ", where estimated $E L P=A C D+1 / 2$ (cataract lens thickness). Further studies to evaluate this may help improve IOL formulas and minimise RS.

We also have to consider the range besides the mean RS when comparing different formulas. RS can be myopic or hypermetropic. There is much variation in refractive outcome, and RS can be difficult to predict in eyes that have small or large ACD. Our small study suggests small ACD causing myopic surprise and large ACD causing hypermetropic surprise. However, this is not significant, and the variability in RS means that there may be myopic or hypermetropic RS (which can range up to $\pm 0.75 \mathrm{D}$ ) in refractive outcomes in eyes with small and large ACD. Hence, it may be prudent to select an IOL power that aims for a refractive outcome of -0.5 to $-0.75 \mathrm{D}$ in order to avoid a hypermetropic refractive outcome after cataract surgery.

In conclusion, in eyes with $A C D<2.8 \mathrm{~mm}$, the least RS was found with the Holladay 2 formula, while in eyes with ACD $>3.2 \mathrm{~mm}$, this was found with the Holladay 1. Overall, the Holladay 2 formula was found to have the least postoperative RS across all ACD:AL ratios. The positive linear correlation between ACD and RS was weak. As ELP is known to influence RS, replacing "ACD" in the IOL formulas with a modified parameter tentatively known as "estimated ELP" may improve refractive outcomes. Further studies are needed to elucidate the relationship accurately.

\section{Acknowledgements}

We would like to thank staff members of the clinic for their help in data collection.

\section{References}

1. Engren AL, Behndig A. Anterior chamber depth, intraocular lens position, and refractive outcomes after cataract surgery. J Cataract Refract Surg. 2013;39(4):572-577. doi:10.1016/j. jcrs.2012.11.019.

2. Yanoff M, Duker JS. Ophthalmology. 4th ed. China: Saunders; 2014.

3. Hoffman RS, Vasavada AR, Allen QB, et al. Cataract surgery in the small eye. J Cataract Refract Surg. 2015;41(11):2565-2575. doi:10.1016/j.jcrs.2015.10.008.

4. Muthappan V, Paskowitz D, Kazimierczak A, Jun AS, Ladas J, Kuo IC. Measurement and use of postoperative anterior chamber depth of fellow eye in refractive outcomes. J Cataract Refract Surg. 2015;41(4):778-784. doi:10.1016/j.jcrs.2014.08.034.

5. Abulafia A, Barrett GD, Rotenberg M et al. Intraocular lens power calculation for eyes with an axial length greater than $26.0 \mathrm{~mm}$ : comparison of formulas and methods. J Cataract Refract Surg. 2015;41(3):548-556. doi:10.1016/j.jcrs.2014.06.033.

6. Stephenson M. Refractive surprises after cataract surgery: The best treatment depends on the amount of residual error: Newtown Square: Review of Ophthalmology; 2016. http://www. reviewofophthalmology.com/content/i/2713/c/45992/. Accessed Jan 2016. 
7. Saal WV. Statistical significance of correlations. Oneonta: The State University of New York; 2005. http://www.oneonta.edu/faculty/vomsaaw/w/psy220/files/SignifOfCorrelations.htm. Accessed Feb 2016.

8. Norrby S. Sources of error in intraocular lens power calculation. J Cataract Refract Surg. 2008;34(3):368-376. doi:10.1016/j.jcrs.2007.10.031.

9. Carifi G, Aiello F, Zygoura V, Kopsachilis N, Maurino V. Accuracy of the refractive prediction determined by multiple currently available intraocular lens power calculation formulas in small eyes. 2015;159(3):577-583. doi:10.1016/j.ajo.2014.11.036.

10. Miraftab M, Hashemi H, Fotouhi A, Khabazkhoob M, Rezvan F, Asgari S. Effect of anterior chamber depth on the choice of intraocular lens calculation formula in patients with normal axial length. Middle East Afr J Ophthalmol. 2014;21(4):307-311. doi:10.4103/0974-9233.142266.

11. Olsen $T$, Olesen $H$, Thim K, Corydon L. Prediction of pseudophakic anterior chamber depth with the newer IOL calculation formulas. J Cataract Refract Surg. 1992 May;18(3):280-285.

12. Atchison DA, Markwell EL. Aberrations of emmetropic subjects at different ages. Vision Res. 2008;48(21):2224-2231. doi:10.1016/j.visres.2008.06.023

13. Steinert RF. Cataract surgery. 3rd ed. California: Saunders; 2010.

14. East Valley Ophthalmology. Zeiss IOLMaster Online Users Instruction Manual V.4 - Anterior Chamber Depth. Jena: Carl Zeiss Meditect AG, 2005. http://www.doctor-hill.com/iol-master/acd. htm. Accessed March 2016. 